\title{
Case fatality risk of the SARS-CoV-2 variant of concern B.1.1.7 in England, 16 November to 5 February
}

Daniel J Grint ${ }^{1}$, Kevin Wing ${ }^{1}$, Elizabeth Williamson ${ }^{1}$, Helen I McDonald 1 , Krishnan Bhaskaran ${ }^{1}$, David Evans ${ }^{2}$, Stephen JW Evans $^{1}$, Alex J Walker ${ }^{2}$, George Hickman ${ }^{2}$, Emily Nightingale ${ }^{3}$, Anna Schultze ${ }^{1}$, Christopher T Rentsch ${ }^{1}$, Chris Bates ${ }^{4}$, Jonathan Cockburn ${ }^{4}$, Helen J Curtis ${ }^{2}$, Caroline E Mortonn ${ }^{2}$, Sebastian Bacon ${ }^{2}$, Simon Davy², Angel YS Wong ${ }^{1}$, Amir Mehrkar $^{2}$, Laurie Tomlinson ${ }^{1}$, Ian J Douglas ${ }^{1}$, Rohini Mathur ${ }^{1}$, Paula Blomquist ${ }^{5}$, Brian MacKenna ${ }^{2}$, Peter Ingelsby ${ }^{2}$, Richard Croker ${ }^{2}$, John Parry $^{4}$, Frank Hester ${ }^{4}$, Sam Harper ${ }^{4}$, Nicholas J DeVito ${ }^{2}$, Will Hulme ${ }^{2}$, John Tazare ${ }^{1}$, Ben Goldacre ${ }^{2,6}$, Liam Smeeth ${ }^{1,6}$, Rosalind M Eggo ${ }^{1,6}$

1. Faculty of Epidemiology and Population Health, London School of Hygiene and Tropical Medicine, London, United Kingdom

2. The DataLab, Nuffield Department of Primary Care Health Sciences, University of Oxford, Oxford, United Kingdom

3. Faculty of Public Health and Policy, London School of Hygiene and Tropical Medicine, London, United Kingdom

4. The Phoenix Partnership (TPP), TPP House, Leeds, United Kingdom

5. COVID-19 Outbreak Surveillance Team, Public Health England, London, United Kingdom

6. These authors contributed equally

Correspondence: Daniel Grint (daniel.grint@lshtm.ac.uk)

Citation style for this article:

Grint Daniel J, Wing Kevin, Williamson Elizabeth, McDonald Helen I, Bhaskaran Krishnan, Evans David, Evans Stephen JW, Walker Alex J, Hickman George, Nightingale Emily, Schultze Anna, Rentsch Christopher T, Bates Chris, Cockburn Jonathan, Curtis Helen J, Morton Caroline E, Bacon Sebastian, Davy Simon, Wong Angel YS, Mehrkar Amir, Tomlinson Laurie, Douglas lan J, Mathur Rohini, Blomquist Paula, MacKenna Brian, Ingelsby Peter, Croker Richard, Parry John, Hester Frank, Harper Sam, DeVito Nicholas J, Hulme Will, Tazare John, Goldacre Ben, Smeeth Liam, Eggo Rosalind M. Case fatality risk of the SARS-CoV-2 variant of concern B.1.1.7 in England, 16 November to 5 February. Euro Surveill. 2021;26(11):pii=2100256. https://doi.org/10.2807/1560-7917.ES.2021.26.11.2100256

The SARS-CoV-2 B.1.1.7 variant of concern (VOC) is increasing in prevalence across Europe. Accurate estimation of disease severity associated with this VOC is critical for pandemic planning. We found increased risk of death for VOC compared with non-VOC cases in England (hazard ratio: 1.67; 95\% confidence interval: 1.34-2.09; $p<0.0001$ ). Absolute risk of death by 28 days increased with age and comorbidities. This VOC has potential to spread faster with higher mortality than the pandemic to date

The severe acute respiratory syndrome coronavirus 2 (SARS-CoV-2) variant of concern B.1.1.7 (VOC) was first identified in Kent, United Kingdom (UK) in autumn 2020. Early analysis suggests it is more transmissible than previously circulating forms (non-VOC) [1] It is now the dominant strain throughout the UK and is increasing in prevalence across Europe [2]. Early reports of increased mortality have not included data on individuals' comorbidities, and this information is needed to facilitate pandemic planning.

Certain PCR assays for SARS-CoV-2 do not amplify one of the spike protein gene targets in this VOC. Spike gene target failure (SGTF) is therefore a proxy for VOC identification, with greater than $95 \%$ sensitivity for VOC diagnosis during the period from 16 November to 11 January [3].

Working on behalf of NHS England, we estimate the risk of death following confirmation of SARS-CoV-2 infection in England, comparing infection with VOC to non-VOC, after accounting for demographic factors and comorbidities. The code and configuration of our analysis is available online (github.com/opensafely/ sgtf-cfr-research).

\section{Study population}

Data were drawn from the OpenSAFELY electronic health records secure research platform, covering $40 \%$ of England's population registered with a general practitioner (GP) (see Supplement, part 1). We used linked data from GPS, SARS-CoV-2 testing, vaccination and mortality records (Supplementary Table S1).

We defined as cases those who tested positive for SARS-CoV-2 between 16 November 2020 and 11 January 2021 and followed them until death or 5 February, when follow-up was censored. Vaccinations against SARS-CoV-2 and diagnoses before the study period were exclusion criteria. The SGTF status was known for 184,786 of 441,161 (42\%) people with confirmed SARSCoV-2 infection between 16 November and 11 January (91,775 VOC; 93,011 non-VOC) (Supplementary Table S4). Full details of the design and analysis are available in the protocol (Supplement, part 9). A total of 867 (419 VOC; 448 non-VOC) all-cause deaths occurred before the administrative censor on 5 February 2021.

The exposure groups were similar demographically (Table 1 ). The VOC group was younger with a lower proportion of older cases ( $\geq 80$ years: $0.9 \%$ in the VOC vs $1.6 \%$ in the non-VOC group), with fewer comorbidities 


\section{TABLE 1}

Demographic and clinical characteristics of the study population, SARS-CoV-2 B.1.1.7 fatality risk, England, 16 November 2020-5 February $(\mathrm{n}=184,786)$

\begin{tabular}{|c|c|c|c|c|c|c|}
\hline \multirow{4}{*}{$\begin{array}{l}\text { Total population } \\
\text { Deaths }\end{array}$} & \multicolumn{2}{|c|}{ Total } & \multicolumn{2}{|c|}{ Non-VOC cases } & \multicolumn{2}{|c|}{ VOC cases } \\
\hline & $\mathrm{n}$ & $\%$ & $\mathrm{n}$ & $\%$ & $\mathrm{n}$ & $\%$ \\
\hline & \multicolumn{2}{|c|}{184,786} & \multicolumn{2}{|c|}{91,775} & \multicolumn{2}{|c|}{93,011} \\
\hline & 867 & 0.5 & 448 & 0.5 & 419 & 0.5 \\
\hline \multicolumn{7}{|l|}{ Time to death (days) } \\
\hline Median (IQR) & \multicolumn{2}{|c|}{$13.0(9.0-21.0)$} & \multicolumn{2}{|c|}{$13.0(8.0-22.0)$} & \multicolumn{2}{|c|}{$14.0(9.0-21.0)$} \\
\hline \multicolumn{7}{|l|}{ Follow-up time } \\
\hline Median (IQR) & \multicolumn{2}{|c|}{$43.0(33.0-60.0)$} & \multicolumn{2}{|c|}{$57.0(40.0-72.0)$} & \multicolumn{2}{|c|}{$36.0(30.0-45.0)$} \\
\hline \multicolumn{7}{|c|}{ Epidemiological week of diagnosis } \\
\hline $16 \mathrm{Nov}-22 \mathrm{Nov}$ & 21,976 & 11.9 & 20,854 & 22.7 & 1,122 & 1.2 \\
\hline 23 Nov-29 Nov & 14,755 & 8.0 & 13,432 & 14.6 & 1,323 & 1.4 \\
\hline 30 Nov-6 Dec & 14,286 & 7.7 & 11,576 & 12.6 & 2,710 & 2.9 \\
\hline $7 \mathrm{Dec}-13 \mathrm{Dec}$ & 18,137 & 9.8 & 11,703 & 12.8 & 6,434 & 6.9 \\
\hline $14 \mathrm{Dec}-20 \mathrm{Dec}$ & 19,963 & 10.8 & 9,043 & 9.9 & 10,920 & 11.7 \\
\hline $21 \mathrm{Dec}-27 \mathrm{Dec}$ & 24,422 & 13.2 & 8,246 & 9.0 & 16,176 & 17.4 \\
\hline $28 \mathrm{Dec}-3 \mathrm{Jan}$ & 34,527 & 18.7 & 9,477 & 10.3 & 25,050 & 26.9 \\
\hline 4 Jan-11 Jan & 36,720 & 19.9 & 7,444 & 8.1 & 29,276 & 31.5 \\
\hline \multicolumn{7}{|l|}{ Sex } \\
\hline Female & 98,099 & 53.1 & 49,468 & 53.9 & 48,631 & 52.3 \\
\hline Male & 86,687 & 46.9 & 42,307 & 46.1 & 44,380 & 47.7 \\
\hline \multicolumn{7}{|l|}{ Age group (years) } \\
\hline $0-<18$ & 27,228 & $14 \cdot 7$ & 14,310 & 15.6 & 12,918 & 13.9 \\
\hline $18-<30$ & 36,969 & 20.0 & 17,302 & 18.9 & 19,667 & 21.1 \\
\hline $30-<40$ & 34,298 & 18.6 & 16,782 & 18.3 & 17,516 & 18.8 \\
\hline $40-<50$ & 32,783 & $17 \cdot 7$ & 15,904 & $17 \cdot 3$ & 16,879 & 18.1 \\
\hline $50-<60$ & 30,484 & 16.5 & 15,261 & 16.6 & 15,223 & 16.4 \\
\hline $60-<70$ & 14,818 & 8.0 & 7,587 & 8.3 & 7,231 & 7.8 \\
\hline $70-<80$ & 5,860 & 3.2 & 3,116 & 3.4 & 2,744 & 3.0 \\
\hline$\geq 80$ & 2,346 & 1.3 & 1,513 & 1.6 & 833 & 0.9 \\
\hline \multicolumn{7}{|l|}{ Ethnicity } \\
\hline White & 105,428 & 57.1 & 52,687 & 57.4 & 52,741 & 56.7 \\
\hline South Asian & 21,562 & 11.7 & 11,880 & 12.9 & 9,682 & 10.4 \\
\hline Black & 4,530 & 2.5 & 1,753 & 1.9 & 2,777 & 3.0 \\
\hline Mixed & 2,628 & 1.4 & 1,175 & 1.3 & 1,453 & 1.6 \\
\hline Other & 2,974 & 1.6 & 1,351 & 1.5 & 1,623 & 1.7 \\
\hline Missing & 47,664 & 25.8 & 22,929 & 25.0 & 24,735 & 26.6 \\
\hline \multicolumn{7}{|c|}{ Categorical number of comorbidities ${ }^{a}$} \\
\hline 0 & 158,017 & $85 \cdot 5$ & 77,538 & $84 \cdot 5$ & 80,479 & 86.5 \\
\hline 1 & 20,606 & 11.2 & 10,768 & 11.7 & 9,838 & 10.6 \\
\hline$\geq 2$ & 6,163 & $3 \cdot 3$ & 3,469 & 3.8 & 2,694 & 2.9 \\
\hline \multicolumn{7}{|c|}{ Index of multiple deprivation quintile } \\
\hline 1 least deprived & 36,560 & 19.8 & 15,973 & $17 \cdot 4$ & 20,587 & 22.1 \\
\hline 2 & 34,767 & 18.8 & 16,000 & $17 \cdot 4$ & 18,767 & 20.2 \\
\hline 3 & 35,181 & 19.0 & 16,192 & 17.6 & 18,989 & 20.4 \\
\hline 4 & 38,603 & 20.9 & 19,479 & 21.2 & 19,124 & 20.6 \\
\hline 5 most deprived & 39,675 & 21.5 & 24,131 & 26.3 & 15,544 & 16.7 \\
\hline
\end{tabular}

a Comorbidities as defined in Supplementary Table S2.

A full table including all factors adjusted for is given in Supplementary Table S3. 


\section{FIGURE 1}

Hazard ratios for death following diagnosis of infection with SARS-CoV-2 VOC vs non-VOC, England, 16 November $2020-5$ February $(\mathrm{n}=184,786)$

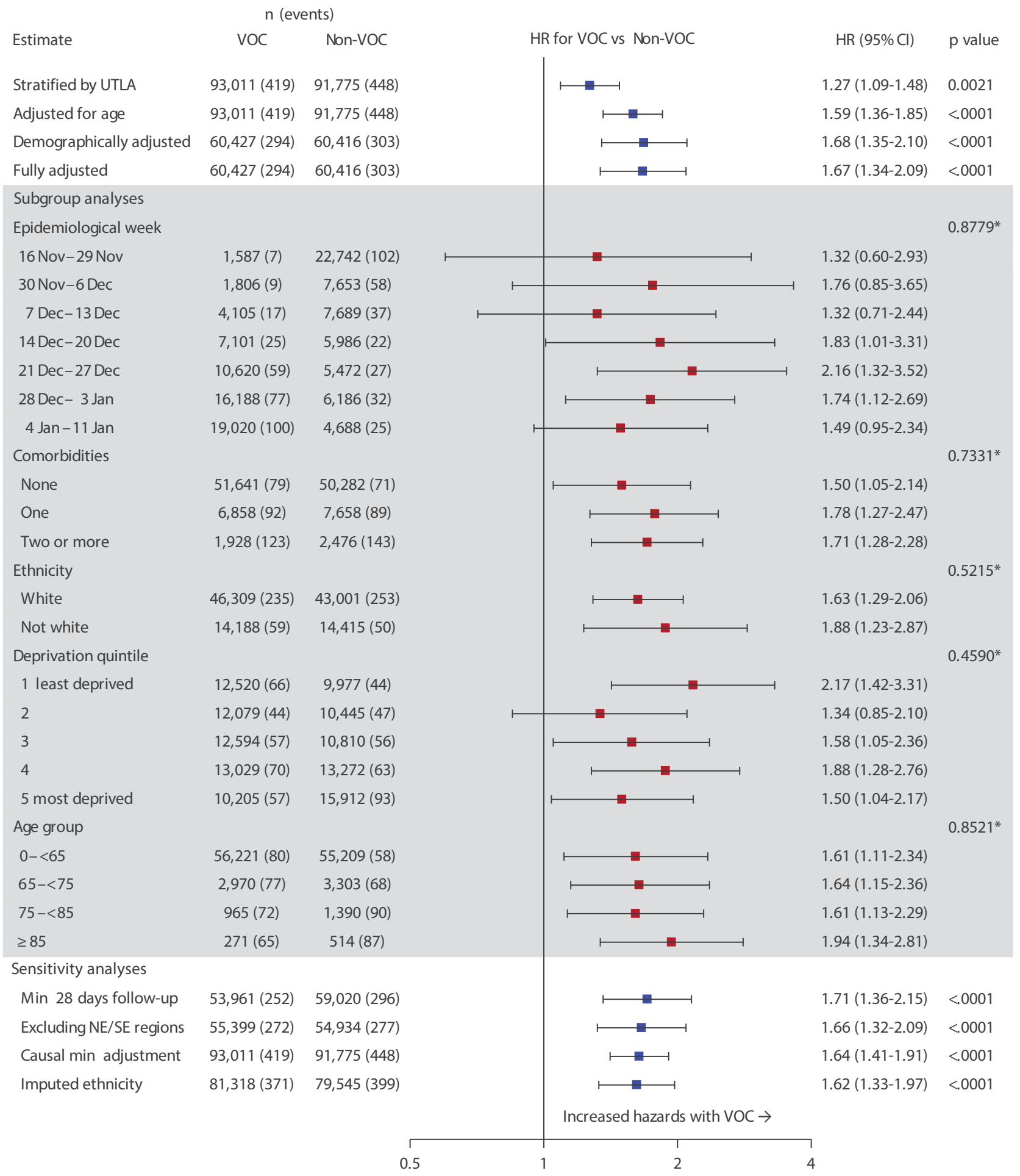

HR: hazard ratio; IMD: index of multiple deprivation; NE: North East; SE: South East; UTLA: upper tier local authority; VOC: variant of concern.

*Likelihood ratio test for interaction between exposure group (VOC, non-VOC) and subgroup.

Cox proportional hazards regression; all models are stratified on region by UTLA; estimating a separate baseline hazard function for each UTLA, with model parameters estimated by maximum likelihood over the full study population.

The demographically adjusted model includes adjustment for: age, sex, IMD, ethnicity, household size, rural urban classification, epidemiological week and care home status. The fully adjusted model includes adjustment for: age, sex, IMD, ethnicity, smoking status, obesity, household size, rural urban classification, comorbidities, epidemiological week and care home status. There was no evidence of non-proportional hazards in this model (global test of Schoenfeld residuals, $p=0.19$ ). The first sensitivity analysis is restricted to people with a minimum of 28 days from testing positive for SARS-CoV-2 to the follow-up censor. The SE and NE NHS England regions are excluded from the second sensitivity analysis (for details see Supplement part 7). The causal minimum adjustment set includes adjustment for: age, care home status, comorbidities, IMD and smoking status.

Missing ethnicity data was imputed for the final listed sensitivity analysis. All subgroup analyses were performed on the fully adjusted model. 
TABLE 2

Absolute risk of death by 28 days, SARS-CoV-2 VOC vs non-VOC infection, England, 16 November 2020-5 February $(\mathrm{n}=112,979)$

\begin{tabular}{|c|c|c|c|c|c|}
\hline \multirow{2}{*}{ Sex } & \multirow{2}{*}{ Age group (years) } & \multicolumn{2}{|c|}{ Non-VOC } & \multicolumn{2}{|c|}{ VOC } \\
\hline & & $\%$ & $95 \% \mathrm{Cl}$ & $\%$ & $95 \% \mathrm{Cl}$ \\
\hline \multicolumn{6}{|c|}{ No comorbidities } \\
\hline \multirow{4}{*}{$\begin{array}{l}\text { Female } \\
n=52,718\end{array}$} & $0-<65$ & 0.05 & $0.03-0.06$ & 0.07 & $0.06-0.09$ \\
\hline & $65-<75$ & 0.45 & $0.30-0.59$ & 0.72 & $0.50-0.95$ \\
\hline & $75^{-<85}$ & 1.08 & $0.71-1.45$ & 1.73 & $1.15-2.31$ \\
\hline & $\geq 85$ & 2.36 & $1.47-3.25$ & 3.75 & $2.34-5.16$ \\
\hline \multirow{4}{*}{$\begin{array}{l}\text { Male } \\
n=42,724\end{array}$} & $0-<65$ & 0.09 & $0.07-0.11$ & 0.14 & $0.11-0.17$ \\
\hline & $65-<75$ & 0.85 & $0.59-1.12$ & 1.37 & $0.96-1.77$ \\
\hline & $75^{-<85}$ & 2.03 & $1.35-2.71$ & 3.24 & $2.19-4 \cdot 30$ \\
\hline & $\geq 85$ & $4 \cdot 38$ & $2.72-6.03$ & 6.87 & $4.33-9.42$ \\
\hline \multicolumn{6}{|c|}{ One comorbidity } \\
\hline \multirow{4}{*}{$\begin{array}{l}\text { Female } \\
n=6,858\end{array}$} & $0-<65$ & 0.11 & $0.08-0.15$ & 0.18 & $0.13-0.24$ \\
\hline & $65-<75$ & 1.09 & $0.78-1.41$ & 1.75 & $1.25-2.25$ \\
\hline & $75^{-<85}$ & 2.60 & $1.84-3.35$ & 4.13 & $2.94-5.32$ \\
\hline & $\geq 85$ & $5 \cdot 54$ & $3.77-7.31$ & 8.64 & $5.91-11.38$ \\
\hline \multirow{4}{*}{$\begin{array}{l}\text { Male } \\
n=6,661\end{array}$} & $0-<65$ & 0.22 & $0.15-0.28$ & 0.35 & $0.25-0.45$ \\
\hline & $65-<75$ & 2.06 & $1.51-2.62$ & 3.29 & $2.44-4.14$ \\
\hline & $75^{-<85}$ & 4.81 & $3.48-6.14$ & $7 \cdot 54$ & $5 \cdot 52-9 \cdot 55$ \\
\hline & $\geq 85$ & 9.94 & $6.87-13.01$ & 15.10 & $10.63-19.58$ \\
\hline \multicolumn{6}{|c|}{ Two or more comorbidities } \\
\hline \multirow{4}{*}{$\begin{array}{l}\text { Female } \\
n=1,921\end{array}$} & $0-<65$ & 0.21 & $0.14-0.28$ & 0.34 & $0.22-0.45$ \\
\hline & $65-<75$ & 1.99 & $1.41-2.57$ & 3.18 & $2.27-4.09$ \\
\hline & $75^{-<85}$ & 4.66 & $3.45-5.87$ & $7 \cdot 31$ & $5.42-9.20$ \\
\hline & $\geq 85$ & 9.65 & $7.01-12.29$ & 14.68 & $10.73-18.63$ \\
\hline \multirow{4}{*}{$\begin{array}{l}\text { Male } \\
n=2,097\end{array}$} & $0-<65$ & 0.40 & $0.27-0.52$ & 0.64 & $0.44-0.84$ \\
\hline & $65-<75$ & 3.72 & $2.74-4.69$ & 5.87 & $4.38-7.35$ \\
\hline & $75^{-<85}$ & 8.44 & $6.44-10.44$ & 12.93 & $9.99-15.87$ \\
\hline & $\geq 85$ & 16.65 & $12.42-20.88$ & 24.34 & $18.55-30.13$ \\
\hline
\end{tabular}

Absolute risk is calculated from the marginal means of a fully adjusted logistic regression model with outcome death by 28 days after positive test for SARS-CoV-2, restricted to the population with a minimum of 28 days from testing to the follow-up censor. Deaths beyond 28 days were censored. The fully adjusted model includes adjustment for: age, sex, index of multiple deprivation, ethnicity, smoking status, obesity, household size, NHS England region, rural/urban classification, comorbidities, epidemiological week and care home status.

(two or more comorbidities: $2.9 \%$ vs $3.8 \%$ ). Non-VOC cases were more frequent in the first 4 weeks of the study period, while VOC cases predominated thereafter. Consequently, median follow-up time was shorter among the VOC group (36 days; interquartile range (IQR): $30-45$ ) than the non-VOC group (57 days; IQR: 40-72).

\section{Relative hazard of death}

We calculated the relative hazard of death for VOC compared with non-VOC cases using a Cox proportional hazards regression model stratified by region (upper tier local authority area (UTLA)) [4,5]. Follow-up began at the date of testing positive for SARS-CoV-2 and was censored on 5 February 2021 or 7 days before receipt of a SARS-CoV-2 vaccine, whichever came first. The 7 days prior to vaccination were censored in this analysis to remove a potential immortal time bias because illness which may lead to death would exclude the booking of and administration of a vaccine. Infection with the VOC was consistently associated with an increased hazard of death. In a fully adjusted analysis accounting for demographics and comorbidities, hazards were twothirds higher in the VOC group (hazard ratio (HR): 1.67; 95\% confidence interval (Cl): 1.34-2.09; $\mathrm{p}<0.0001)$ compared with non-VOC (Figure 1). Increased hazards for $V O C$ were consistent across all pre-specified subgroup analyses including epidemiological week, age group, categorical number of comorbidities, ethnicity and index of multiple deprivation (IMD) quintile [6]. Increased hazards were also consistent across all prespecified sensitivity analyses; in an analysis restricted to people testing positive for SARS-CoV-2 infection a minimum of 28 days before the censoring date, the hazard ratio was 1.71 (95\% Cl: 1.36-2.15; $\mathrm{p}<0.0001)$.

\section{Absolute risk of death by 28 days}

We found a consistently higher absolute risk of death by 28 days after a SARS-CoV-2-positive test in all groups stratified by age, sex and presence of comorbidities in 
Excess risk of death by 28 days, SARS-CoV-2 VOC compared with non-VOC infection, England, 16 November 2020-5 February $(\mathrm{n}=112,979)$

\section{A. Males}

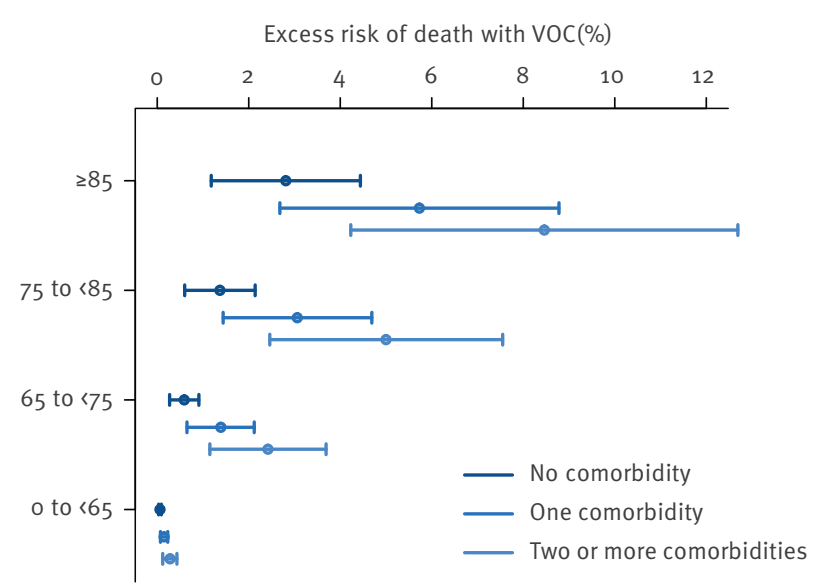

B. Females

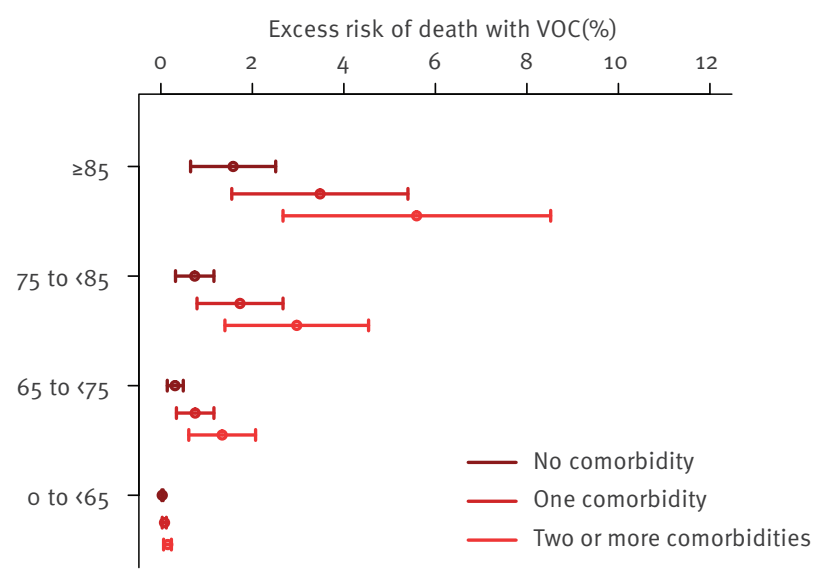

VOC: variant of concern.

The figure shows risk difference of death by 28 days for VOC compared with non-VOC, with $95 \%$ confidence interval.

VOC, compared with non-VOC (Table 2). Risk of death was estimated by the marginal means of a fully adjusted logistic regression model. This analysis was restricted to 112,979 people diagnosed with SARS-CoV-2 a minimum of 28 days before the censoring date, with the outcome death by 28 days after a positive test. Deaths occurring beyond 28 days were censored. Data were not censored 7 days prior to vaccination in this analysis as vaccination is contraindicated in the month following a positive SARS-CoV-2 test. Consistent with the Cox model above, VOC was associated with increased odds of death in this model (adjusted odds ratio (AOR): 1.73; 95\% Cl: 1.34-2.23; $p$ value<0.0001, vs non-VOC). The risk of death was low for people younger than 65 years in the absence of comorbidities; in this age group it was higher for male than female cases (VOC: males: $0.14 \%$; females: $0.07 \%$ vs non-VOC: males: $0.09 \%$; females: $0.05 \%)$. The risk of death was consistently higher for male cases and increased with age and the presence of comorbidities. The highest risk of death within 28 days was seen among those 85 years and older with two or more comorbidities: VOC: males $24.3 \%$; females: $14.7 \%$; non-VOC: males: $16.7 \%$; females: $9.7 \%$ ). The excess risk of death within 28 days for VOC compared with non-VOC is shown in Figure 2.

\section{Discussion}

The SARS-CoV-2 B.1.1.7 VOC has been the subject of intense research since its emergence. Increased transmissibility means it is now the most common variant in the UK, a trend confirmed here. We found that this VOC was associated with two-thirds higher case fatality than the previously circulating virus in this unvaccinated population. For every three deaths in a population with the previously circulating virus we would expect five deaths in a similar population with VOC. Other studies have assessed the relative mortality of the VOC with similar conclusions [7-10], however, our results are the first to include detailed information on the presence of comorbidities. Interestingly, the effects of age and comorbidities appear to be collinear as adjustment for comorbidities did not alter the findings after adjustment for age. As prevalence of many comorbidities is associated with age, this finding appears plausible [11]. The consistency of the effect for each epidemiological week of diagnosis shows that the increase in mortality due to VOC could not be explained by other secular changes in mortality such as hospitals exceeding capacity.

The absolute risks of death by 28 days demonstrated an increasing risk with age and presence of comorbidities; male cases had a consistently higher risk of death than female cases. However, age and comorbidity risk factors associated with poor non-VOC outcomes appear to be similar to those with this VOC. Therefore, prioritisation for vaccination and shielding can remain the same.

In the UK, all-cause death by 28 days after confirmation of SARS-CoV-2 infection is the standard definition of SARS-CoV-2 mortality [12], so we used death from any cause as the primary outcome. In a sensitivity analysis restricted to people diagnosed with SARSCoV-2, a minimum of 28 days before the censoring date and logistic regression with deaths censored beyond 28 days, the results were consistent.

This analysis includes people testing positive for SARSCoV-2. People with asymptomatic or mild infection may 
not present for testing. Consequently, our estimates of absolute risk of death by 28 days may be overestimates of the true case fatality ratio. In addition, SARS-CoV-2 tests performed in hospital settings in the UK are not tested for PCR S-gene target failure and are therefore not included.

This VOC is now prevalent across Europe and is likely to become the most frequent variant following the pattern seen in the UK [2]. Policymakers and pandemic planners need to account for higher mortality of this VOC. Crucially, emerging data suggest that the currently approved vaccines for SARS-CoV-2 are effective against the B.1.1.7 VOC [13]. This study highlights the importance of robust national vaccination programmes and infection control measures to contain the SARS-CoV-2 pandemic. Unmitigated spread of the B.1.1.7 VOC has the potential to be both faster and more deadly than the pandemic to date.

\section{Ethical approval}

This study was approved by the Health Research Authority (REC reference 20/LO/0651) and by the LSHTM Ethics Board (reference 21863).

\section{Acknowledgements}

We are grateful for the support received from the TPP Technical Operations team and for generous assistance from the information governance and database teams at NHS England/NHSX. We would also like to thank PHE for making SARS-CoV-2 testing data available.

Funding: This work was supported by the Medical Research Council MR/V015737/1. TPP provided technical expertise and infrastructure within their data centre pro bono in the context of a national emergency.

Rosalind Eggo is funded by HDR UK (grant: MR/So03975/1), MRC (grant: MC_PC 19065), NIHR (grant: NIHR200908).

\section{Conflict of interest}

None declared.

\section{Authors' contributions}

DJG, RME, and KW led the study. RME conceived the study. DJG, RME, KW, KB, CTR, and LS drafted the study protocol. DJG, KW, RME, EW, HIMCD, KB, DE, SJWE, AJW, EN, AS, CTR, HJC, CEM, AYSW, RM, PB, WH, and JT contributed to data preparation and variable definitions. $\mathrm{DE}, \mathrm{GH}, \mathrm{CB}, \mathrm{JC}, \mathrm{HJC}$, CEM, SB, SD, AM, LT, IJD, BMacK, PI, RC, JP, FH, SH, NJDeV, $W H, B G$, and $L S$ contributed to building the analytical platform. DJG, KW, RME, KB, SJWE, and LS contributed to study design. DJG performed the statistical analysis. All authors contributed to manuscript preparation and refinement. 2021;eabg3055. https://doi.org/10.1126/science.abg3055 PMID: 33658326

2. European Centre for Disease Prevention and Control (ECDC). SARS-CoV-2 - increased circulation of variants of concern and vaccine rollout in the EU/EEA,14th update. Stockholm. ECDC; 2021. Available from: https://www.ecdc.europa.eu/ sites/default/files/documents/RRA-covid-19-14th-update-15feb-2021.pdf

3. Public Health England (PHE). Investigation of novel SARS-CoV-2 variant. Variant of concern 20201/01. London: PHE. [Accessed: 25 Feb 2021]. Available from: https://assets.publishing. service.gov.uk/government/uploads/system/uploads/ attachment_data/file/959438/Technical_Briefing_VOC_SH_ NJL2_SH2.pdf

4. Cox DR. Regression models and life 1972;34(2):187-202. https://doi.org/10.1111/j.2517-6161.1972. tboo899.x

5. Cox DR, Oakes D. Analysis of survival data. London: CRC press; 1984. ISBN 9780412244902.

6. Ministry of Housing, Communities Local Government. The English indices of deprivation 2019 (IoD2019). London: Ministry of Housing, Communities Local Government; 2019. Available from: https://www.gov.uk/government/statistics/ english-indices-of-deprivation-2019

7. NERVTAG. NERVTAG paper on COVID-19 variant of concern B.1.1.7: Paper from the New and Emerging Respiratory Virus Threats Advisory Group (NERVTAG) on new coronavirus (COVID-19) variant B.1.1.7. London: Department of Health and Social Care; 2021. Available from: https://www.gov.uk/government/publications/ nervtag-paper-on-covid-19-variant-of-concern-b117

8. Iacobucci G. Covid-19: New UK variant may be linked to increased death rate, early data indicate. BMJ. 2021;372(230):n230. https://doi.org/10.1136/bmj.n230 PMID: 33500262

9. Davies NG, Jarvis CI, CMMID COVID-19 Working Group, Edmunds WJ, Jewell NP, Diaz-Ordaz K, Keogh RH, et al. Increased mortality in community-tested cases of SARS-CoV-2 lineage B.1.1.7. Nature. 2021. https://doi.org/10.1038/s41586021-03426-1 PMID: 33723411

10. Challen R, Brooks-Pollock E, Read JM, Dyson L, TsanevaAtanasova K, Danon L. Risk of mortality in patients infected with SARS-CoV-2 variant of concern 202012/1: matched cohort study. BMJ. 2021;372(579):n579. https://doi.org/10.1136/bmj. n579 PMID: 33687922

11. Walker JL, Grint DJ, Strongman H, Eggo RM, Peppa M, Minassian C, et al. UK prevalence of underlying conditions which increase the risk of severe COVID-19 disease: a point prevalence study using electronic health records. BMC Public Health. 2021;21(1):484. https://doi.org/10.1186/s12889-02110427-2 PMID: 33706738

12. Department of Health and Social Care. New UK-wide methodology agreed to record COVID-19 deaths. London: Department of Health and Social Care; 2020. Available from: https://www.gov.uk/government/news/ new-uk-wide-methodology-agreed-to-record-covid-19-deaths

13. Public Health England (PHE). PHE monitoring of the effectiveness of COVID-19 vaccination. London: PHE; 2021 Available from: https://www.gov.uk/government/publications/ phe-monitoring-of-the-effectiveness-of-covid-19-vaccination

\section{License, supplementary material and copyright}

This is an open-access article distributed under the terms of the Creative Commons Attribution (CC BY 4.0) Licence. You may share and adapt the material, but must give appropriate credit to the source, provide a link to the licence and indicate if changes were made.

Any supplementary material referenced in the article can be found in the online version.

This article is copyright of the authors or their affiliated institutions, 2021.

\section{References}

1. Davies NG, Abbott S, Barnard RC, Jarvis Cl, Kucharski

AJ, Munday JD, et al. Estimated transmissibility and impact of SARS-CoV-2 lineage B.1.1.7 in England. Science. 